\title{
GMR
}

\section{Identification of key biomarkers involved in osteosarcoma using altered modules}

\author{
Z.Z. Liu, S.T. Cui, B. Tang, Z.Z. Wang and Z.X. Luan \\ Department of Orthopaedics, the Second People's Hospital of Liaocheng, \\ Linqing, China \\ Corresponding author: Z.Z. Liu \\ E-mail: liuzizhenwz@163.com
}

Genet. Mol. Res. 15 (3): gmr. 15038277

Received December 15, 2015

Accepted February 11, 2016

Published August 26, 2016

DOI http://dx.doi.org/10.4238/gmr.15038277

Copyright (C) 2016 The Authors. This is an open-access article distributed under the terms of the Creative Commons Attribution ShareAlike (CC BY-SA) 4.0 License.

\begin{abstract}
The aim of this study was to screen for key biomarkers of osteosarcoma (OS) by tracking altered modules. Protein-protein interaction (PPI) networks of OS and normal groups were constructed and re-weighted using the Pearson correlation coefficient (PCC), respectively. The condition-specific modules were explored from OS and normal PPI networks using a clique-merging algorithm. Altered modules were identified by a maximum weight bipartite-matching method. The important biological pathways in OS were identified by a pathway-enrichment analysis using genes from disrupted modules. The most important genes in these pathways were selected as key biomarkers. Finally, the mRNA and protein expressions of hub genes in OS bone tissues were analyzed using reverse transcription-polymerase chain reaction and western blotting, respectively. We identified 703 and 2270 modules in normal and disease networks, respectively; 150 altered modules were identified from among these and explored. We identified 10 important pathways based on gene pairs with altered PCC $>1$ in the disrupted modules $(\mathrm{P}<0.01)$, and PCNA, ATP6V1C2, ATP6V1G3,
\end{abstract}


FEN1, CDC7, and RPA3 (expressed in these pathways) were selected as key genes of OS. We observed that these genes (and the proteins they encoded) were differentially expressed between normal and OS samples $(\mathrm{P}<0.01)$ (excluding ATP6V1C2, whose protein expression did not differ significantly). Therefore, we identified 5 gene signatures that may be potential biomarkers for the detection and effective therapy of OS.

Key words: Osteosarcoma; Altered modules; Key genes; Reverse transcription-polymerase chain reaction; Western blot

\section{INTRODUCTION}

Osteosarcoma (OS) is a cancerous tumor occurring in the bone. It is the most common histological form of primary bone cancer (Ottaviani and Jaffe, 2009) and occurs mostly in adolescents where the proliferation is most active, with a second peak in patients older than 40 years (Anninga, 2013). It is characterized by cells with OS differentiation, poorly defined borders, and cortical destruction that results in bone pain and compromised structural integrity (Luetke et al., 2014). Approximately $30 \%$ of the patients with OS present clinically detectable metastatic disease at diagnosis (Kaste et al., 1999; Kager et al., 2003). So far, researchers have not thoroughly investigated treatment methods that could significantly increase survival, excluding preoperative chemotherapy (Buddingh et al., 2011).

Recent studies have mainly focused on individual gene-related analyses (Rosemann et al., 2014; Liu and Chen, 2015; Zeng and Xu, 2015; Li et al., 2016). However, these do not accurately reflect the interaction between integral genes under different conditions (Slonim, 2002). Fortunately, functional modules integrate the most closely related proteins or genes through their interactions. Recently, Srihari and Ragan (2013) reported a systematic method to track the behavior of genes and modules across normal and disease conditions, in order to identify and elucidate significant pathways.

In this study, we systematically tracked the altered modules of reweighted proteinprotein interaction (PPI) networks in order to identify the key biomarkers of OS. Initially, we inferred the PPI networks of normal and OS systems, based on the Pearson correlation coefficient (PCC). Subsequently, the modules from PPI networks were identified using a clique-merging algorithm. We then extracted the altered modules according to the module correlation density (MCD). We also analyzed the active genes and the functional pathways of gene pairs with a PCC $>1$ in disrupted modules. Ultimately, the expression of key genes was verified by reverse transcription-polymerase chain reaction (RT-PCR) and western blot analyses. The results of this study could elucidate the mechanism of OS, which would contribute to its diagnosis and treatment.

\section{MATERIAL AND METHODS}

\section{Data collection and preprocessing}

\section{Microarray expression data}

The gene expression profile E-MEXP-3628 (Jones et al., 2012) comprising 14 OS

Genetics and Molecular Research 15 (3): gmr.15038277 
samples and 4 normal bone samples was downloaded onto the A-AFFY-44-Affymetrix GeneChip Human Genome U133 Plus 2.0 platform (Affymetrix; Life Technologies, Carlsbad, CA, USA). The gene profile data were preprocessed using the express function in the Affy package (Gautier et al., 2004). Background adjustments were performed using a robust multiarray average method. Gene expression was summarized using median polish. Ultimately, 20,102 genes were obtained.

\section{PPI network data}

All PPIs and their combined-scores (comprising 1,048,576 interactions) were retrieved from the Search Tool for the Retrieval of Interacting Genes (STRING; http://string-db.org/) database (von Mering et al., 2005). The oneself-loops were removed and interactions with combined-scores $\geq 0.8$ were retained for the construction of a PPI sub-network; the completed PPI network was composed of 8590 nodes and 53,975 interactions.

\section{Construction of re-weighted PPI network}

Gene interactions in the PPI sub-networks (occurring under OS and normal conditions) were re-weighted using PCC (value ranging from -1 to +1) (Li et al., 2012). Additionally, we defined the absolute value of PCC of each gene-gene interaction as the value of the interaction in the PPI sub-network. The PCC of a pair of genes ( $m$ and $n$ ), which encoded the corresponding paired interacting proteins ( $u$ and $v$ ) in a PPI network, was defined as:

$$
\text { PCCm, } n=\frac{1}{S-1} \sum_{k=1}^{s}\left(\frac{g(m, k)-\bar{g}(m)}{p(m)}\right) \cdot\left(\frac{g(n, \mathrm{k})-\overline{\mathrm{g}}(n)}{p(n)}\right) \quad \text { (Equation 1) }
$$

where $s$ denotes the number of samples; $g(m, k)$ or $g(n, k)$ indicates the level of expression of gene $\mathrm{m}$ or $\mathrm{n}$ in the sample $\mathrm{k} ; \bar{g}(m)$ or $\bar{g}(n)$ represents the mean expression of gene $\mathrm{m}$ or $\mathrm{n}$; and $\rho(m)$ or $\rho(n)$ denote the standard deviation of expression of gene $m$ or $n$.

In this study, the PCC of each gene-gene interaction was defined by its weight. All interactions with $\mathrm{PCC}$ changes $>1$, i.e. $\left|\mathrm{PCC}(m, n)_{1}-\mathrm{PCC}(m, n)_{2}\right|>1$, between two conditions were selected for further analysis.

\section{Identifying modules in PPI sub-networks}

Modules were identified in PPI networks using the clique-merging method (Liu et al., 2009; Srihari and Leong, 2013). All maximal cliques were obtained from the disease and normal networks using the fast depth-first method; the cliques were ranked according to their weighted density; highly overlapping cliques were then either merged or removed. The score of a clique (C) was defined as its weighted density:

$$
\operatorname{score}(C)=\frac{\sum_{u \in c, v \in c} w(u, v)}{|C| \cdot(|C|-1)}
$$

where $w(u, v)$ denoted the weight of interactions between $u$ and $v$.

Highly overlapping maximal cliques were removed, or were merged into single bigger

Genetics and Molecular Research 15 (3): gmr.15038277 
cliques. We also calculated the interconnectivity of cliques to determine if two overlapped cliques should be merged together. The inter-connectivity between the non-overlapping portions of cliques $\mathrm{C}_{1}$ and $\mathrm{C}_{2}$ was defined as follows:

$$
\text { inter-score }\left(C_{i,} C_{j}\right)=\sqrt{\frac{\sum_{u \epsilon}\left(c_{i}-c_{j}\right) \Sigma_{v \in c j} w(u, v)}{\left|C_{i}-C_{j}\right| \cdot\left|C_{j}\right|} \cdot \frac{\sum_{u \epsilon}\left(c_{j}-c_{i}\right) \Sigma_{v \in c_{i}} w(u, u)}{\left|C_{j}-C_{i}\right| \cdot\left|C_{i}\right|}} \quad \text { (Equation 3) }
$$

Given a set of cliques ranked in descending order of the score $\left\{C_{1}, C_{2}, \ldots, C_{k}\right\}$, if $\left|C_{i} \cap C_{j}\right| / \mid C_{j}$ $\mid>t_{0}$, the weighted inter-connecting scores between $C_{i}$ and $C_{j}$ were analyzed. $C_{i}$ and $C_{j}$ were combined into the module if the inter-score was $>t_{\mathrm{m}}$ (Srihari and Ragan, 2013). In this study, $\mathrm{t}_{0}=0.5$ and $\mathrm{t}_{\mathrm{m}}=0.25$.

\section{Identification of altered modules}

If $S=\left\{S_{1}, S_{2}, \ldots, S_{\mathrm{n}}\right\}$ and $T=\left\{T_{1}, T_{2}, \ldots, T_{\mathrm{m}}\right\}$ are the sets of modules identified from the normal and disease networks, respectively, for each $S_{i} \in S$, the MCD was measured as follows:

$$
d_{c c}\left(S_{i}\right)=\frac{\Sigma_{p, q \in S_{i}} P C C((p, q), N)}{\left(\frac{\left|S_{i}\right|}{2}\right)}
$$

where $p$ and $q$ denote a pair of genes encoding the corresponding paired proteins. The correlation densities in the disease modules were calculated in a similar manner.

We built a similarity graph $M=\left(V_{M}, E_{M}\right)$, where $V_{M}=\{S \cup T\}$ and $E_{M}=\left\{\left(S_{i}, T_{j}\right): J\left(S_{i}\right.\right.$, $\left.\left.T_{j}\right) \geq t_{j}, \Delta_{C C}\left(S_{i}, T_{j}\right) \geq \delta\right\}$, wherein $J\left(S_{i}, T_{j}\right)=\left|S_{i} \cap T_{j} /\right| S_{i} \cup T_{j} \mid$ indicates the Jaccard similarity and $\Delta_{\mathrm{CC}}\left(S_{i}, T_{j}\right)=\left|d_{c c}\left(S_{i}\right)-d_{c c}\left(T_{j}\right)\right|$ denotes the differential correlation density between $S_{i}$ and $T_{j}$ (Srihari and Ragan, 2013). Furthermore, the disrupted module pairs T $\left(S_{i}, T_{j}\right)$ were identified by determining the maximum weight matching in $\mathrm{M}$; these were then ranked in descending order of their differential density $\Delta_{C C}$. The disrupted module pairs with $t_{J} \geq 2 / 3$ and $\Delta_{C C} \geq 0.05$ were defined as distinct modules.

\section{Pathway-enrichment analysis}

Database for Annotation, Visualization, and Integrated Discovery (DAVID) is a webaccessible program that offers a number of data-mining tools used to determine the biological functional annotations for thousands of genes (Huang et al., 2009). In this study, the KEGG pathway-enrichment analysis was performed to further explore the biological functions of genes in disrupted modules and gene pairs with PCC $>1$, using DAVID. The top 10 pathways (ascending order) with $\mathrm{P}<0.01$ were denoted as important pathways.

\section{Screening key genes}

The gene compositions of each module were analyzed to better understand the differences of altered modules. The key genes in altered modules, including missed genes (genes in normal module, but not in the disease module), added genes (genes in the disease module, but not in the normal module), and intersection genes, were analyzed. In this study, we focused mainly on the added genes, missed genes, and genes in key pathways.

Genetics and Molecular Research 15 (3): gmr.15038277 


\section{RT-PCR and western blot validation}

In order to verify key genes identified from our study, 10 OS samples and 10 normal bone tissues were selected to perform verification analysis. The total RNA was extracted using the Trizol reagent (Invitrogen, Carlsbad, CA, USA) in accordance with the manufacturer instructions and utilized in first-strand synthesis. RT-PCR was performed using primer sequences specific for 6 hub genes (PCNA, ATP6V1C2, ATP6V1G3, FEN1, CDC7, and RPA3), as shown in Table 1 . The PCR mixture was composed of $10 \mu \mathrm{L} 10 \mathrm{X}$ PCR Buffer, 1 $\mu \mathrm{L}$ Taq DNA polymerase, $3 \mu \mathrm{L}$ forward and reverse primer each, and $8 \mu \mathrm{L}$ dNTPs. The PCR conditions were set as follows: initial denaturation at $95^{\circ} \mathrm{C}$ for $3 \mathrm{~min} ; 30$ cycles of denaturation at $95^{\circ} \mathrm{C}$ for $40 \mathrm{~s}$, annealing at $58^{\circ} \mathrm{C}$ for $30 \mathrm{~s}$, and extension at $72^{\circ} \mathrm{C}$ for $45 \mathrm{~s}$; and a final elongation at $72^{\circ} \mathrm{C}$ for $7 \mathrm{~min}$. The synthesized cDNA was used as the template, and $\beta$-actin was used as the internal reference.

Table 1. Primer sequences and product lengths of the six hub genes.

\begin{tabular}{|c|c|c|}
\hline Genes & Primer sequences $\left(5^{\prime} \rightarrow 3^{\prime}\right)$ & Length (bp) \\
\hline \multirow[t]{2}{*}{$P C N A$} & F: CAAGTAATGTCGATAAAGAGGAGG & \multirow[t]{2}{*}{126} \\
\hline & R: GTGTCACCGTTGAAGAGAGTGG & \\
\hline \multirow[t]{2}{*}{ ATP6VIC2 } & F: GCCGCCTACAACACTCTGAAGA & \multirow[t]{2}{*}{145} \\
\hline & R: CGATGACCAGAAGTGTGACGAG & \\
\hline \multirow{2}{*}{$\overline{A T P 6 V 1 G 3}$} & F: GCGATTGAAGCAAGCCAAGGAG & \multirow[t]{2}{*}{122} \\
\hline & R: CATCTGAGAGATTATTCTGAGAGC & \\
\hline \multirow[t]{2}{*}{ FEN1 } & F: ACTAAGCGGCTGGTGAAGGTCA & \multirow[t]{2}{*}{146} \\
\hline & R: GCAGCATAGACTTTGCCAGCCT & \\
\hline \multirow[t]{2}{*}{$C D C 7$} & F: GGAAAACTGCCAGTTCTTGCCC & \multirow[t]{2}{*}{150} \\
\hline & R: GGCACTTTGTCAAGACCTCTGG & \\
\hline \multirow[t]{2}{*}{$R P A 3$} & F: AAGCCTGTCTGCTTCGTAGGGA & \multirow[t]{2}{*}{157} \\
\hline & R: CGGTTACTCTTCCAACCACTTCC & \\
\hline \multirow[t]{2}{*}{$\beta$-actin } & F: CTCCATCCTGGCCTCGCTGT & \multirow[t]{2}{*}{268} \\
\hline & R: GCTGTCACCTTCACCGTTCC & \\
\hline
\end{tabular}

All tissue samples were ground to a powder in liquid nitrogen and then incubated with lysis buffer [62.5 mM Tris- $\mathrm{HCl}, \mathrm{pH} 6.8,10 \%$ glycerol, and 2\% sodium dodecyl sulfate (SDS)] on ice. Protein concentration was determined by the Bradford assay (Bio$\mathrm{Rad})$. Ten micrograms of total protein was separated using 12\% SDS-polyacrylamide gel electrophoresis; the separated proteins were electrically transferred at $4^{\circ} \mathrm{C}$ and $300 \mathrm{~mA} \mathrm{(2} \mathrm{h)}$ onto a nitrocellulose (NC) membrane. The NC membrane was then incubated in Tris-buffered saline Tween (TBST) with $5 \%$ skimmed milk at $37^{\circ} \mathrm{C}$ for $2 \mathrm{~h}$; the membrane was blocked with a blocking solution $(1: 10,000)$ at $37^{\circ} \mathrm{C}$ for $2 \mathrm{~h}$, and subsequently incubated with rabbit anti-human IgG antibody (Sigma-Aldrich, St. Louis, MO, USA). Unbound antibodies were washed with TBST (3 times). The membrane was then incubated with horseradish peroxidase (HRP)-labeled sheep anti-rabbit IgG secondary antibody (1:5000; Amersham Pharmacia Biotech, Little Chalfont, Buckinghamshire, UK) for $2 \mathrm{~h}$ at $37^{\circ} \mathrm{C}$. The excess antibodies were washed with TBST, and the reaction was completed by the addition of the HRP substrate for 3 min. The membrane was then analyzed in a darkroom.

\section{Data analysis}

The PCR products were electrophoresed on 1.5\% agarose gel and analyzed using the 
Quantity One software. Gene expression was determined relative to that of $\beta$-actin. The gray value of the target bands obtained by western blot was analyzed using the Image J software. $\mathrm{P}$ values $<0.05$ were considered to be statistically significant.

\section{RESULTS}

\section{Analyzing disruptions in the PPI sub-network}

PPI sub-networks containing 20,102 intersecting genes (identified by pre-processing as well as those in the general PPI network) were constructed. The PPI sub-networks exhibited an equal number of interactions $(49,151)$ and nodes $(8015)$, with mean PCC scores of 0.320 and 0.698 in the OS and normal groups, respectively. The number of interactions in the OS network was higher than that in the normal cell network, at a score distribution of -0.5-0.7 (Figure 1). Furthermore, 10,570 interactions were extracted with an absolute difference of PCC $>1$ between two conditions for further analyses.

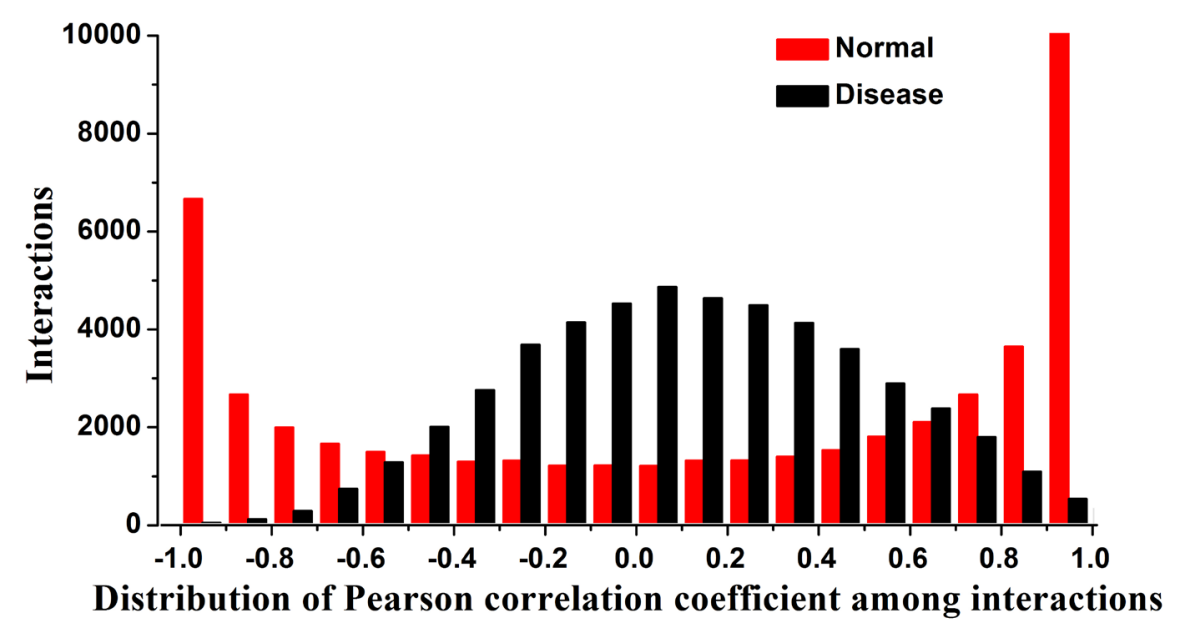

Figure 1. Expression correlation-wise distribution of interactions in osteosarcoma and normal group.

\section{Analysis of disruptions in OS modules}

A total of 8405 maximal cliques were screened in normal and OS PPI sub-networks based on a node threshold-value $\geq 5$. A total of 703 and 2270 modules were extracted from the normal and OS PPI sub-networks, respectively (Table 2). The average module size and maximum MCD of OS were lower than those of the normal bone tissues. Figure 2 shows the relationship between the numbers and weighted densities of modules. The distribution of modules was higher in OS tissues than in normal tissues, at a weighted density of -0.2-0.4.

Subsequently, 150 disrupted module pairs were obtained, with a $t_{J}=2 / 3$ and $\delta=$ 0.05 . These disrupted module pairs were composed of 587 genes. The OS group contained 77 correlation-altered modules more than the normal group, based on the MCD of the modules. 
Table 2. Properties of normal and disease modules.

\begin{tabular}{l|c|c|c|c|c}
\hline Module set & No. of modules & Average module size & \multicolumn{3}{|c}{ Correlation } \\
\cline { 4 - 6 } & & & Max & Min & Average \\
\hline Normal & 703 & 46.8 & 0.8 & -0.2 & 0.1 \\
\hline Disease & 2270 & 21.9 & 0.7 & -0.1 & 0.1 \\
\hline
\end{tabular}

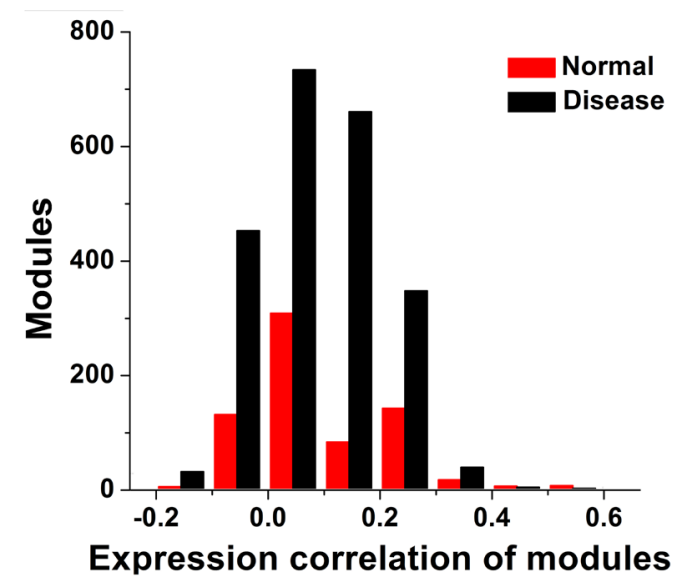

Figure 2. Correlation-wise distribution of modules in osteosarcoma and normal group. Expression correlation of each module is equivalent to the weighted correlation density in the module.

\section{Pathway-enrichment analysis}

The extraction of the intersections of genes in disrupted modules, as well as genes with $\mathrm{PCC}$ changes $>1$, yielded 489 genes, which were enriched in 37 pathways $(\mathrm{P}<0.01)$. The top 10 pathways are summarized in ascending order in Table 3.

Table 3. Ten most significant gene interaction pathways in disrupted modules with Pearson correlation coefficient $>1$.

\begin{tabular}{l|l|c}
\hline KEGG ID & Terms & P value \\
\hline Hsa03050 & Proteasome & $1.5 \mathrm{E}-34$ \\
\hline Hsa04110 & Cell cycle & $2.3 \mathrm{E}-26$ \\
\hline Hsa05120 & Epithelial cell signaling in Helicobacter pylori infection & $1.3 \mathrm{E}-14$ \\
\hline Hsa04114 & Oocyte meiosis & $6.0 \mathrm{E}-10$ \\
\hline Hsa05110 & Vibrio cholerae infection & $6.1 \mathrm{E}-10$ \\
\hline Hsa05222 & Small cell lung cancer & $1.2 \mathrm{E}-9$ \\
\hline Hsa00190 & Oxidative phosphorylation & $1.5 \mathrm{E}-9$ \\
\hline Hsa04510 & Focal adhesion & $9.1 \mathrm{E}-9$ \\
\hline Hsa05200 & Pathways in cancer & $9.2 \mathrm{E}-9$ \\
\hline Hsa03030 & DNA replication & $1.6 \mathrm{E}-8$
\end{tabular}

\section{Screening of key genes}

The 587 genes in the disrupted module pairs contained 211 missed genes, 121 added genes, and 46 intersection genes. Six of the intersection genes played a role in the top 10 significant pathways. In this study, these 6 genes were treated as the key genes involved in OS.

Genetics and Molecular Research 15 (3): gmr.15038277 


\section{RT-PCR and western blot detection}

Relative expressions of the screened genes $(\mathrm{N}=6)$, as determined by RT-PCR and western blot analyses, are presented in Figure 3. The expression of these key genes was significantly different between OS samples and controls $(\mathrm{P}<0.05)$ at the mRNA level (Figure 3A). Additionally, relative protein expressions of PCNA, ATP6V1G3, FEN1, CDC7, and RPA3 were also remarkably different among the OS and normal samples $(\mathrm{P}<0.05)$; however, the difference in the ATP6V1C2 protein expression was not significant ( $P>0.05$; Figure 3B).

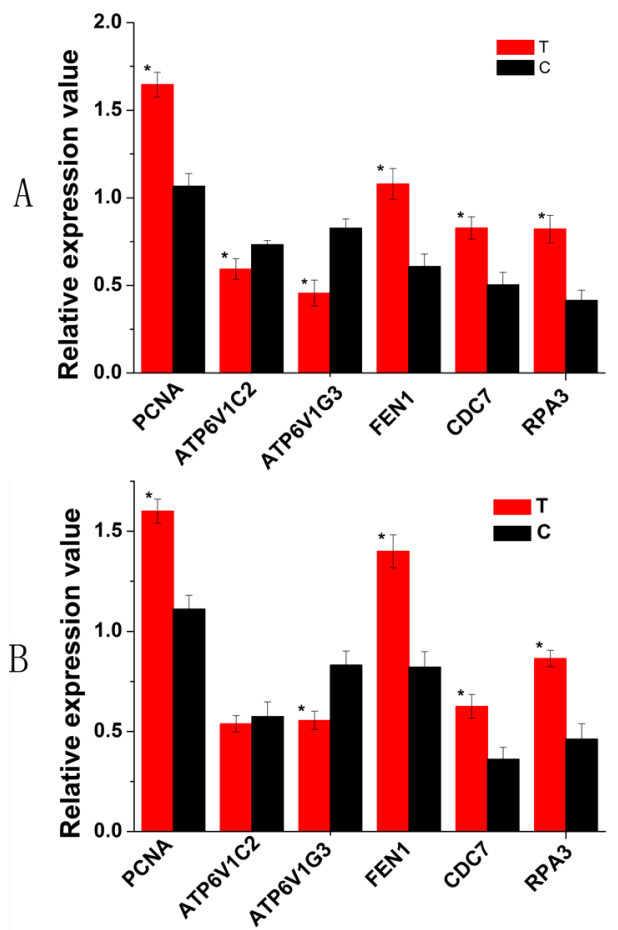

Figure 3. Results of reverse transcription polymerase chain reaction and western blot analysis. A. B. Results of RTPCR and western blot: T represents the osteosarcoma group, $\mathrm{C}$ represents the control group. ${ }^{*}$ Relative expressions of a gene was significant at $\mathrm{P}<0.05$.

\section{DISCUSSION}

The identification of differentially expressed genes under different conditions is a conventional method for the analysis of gene expression data; however, this does not accurately reflect the integral interaction between these genes under different conditions (Slonim, 2002). The rapid advancement in high-throughput experimental methods has led to the identification of a large amount of PPI data, which in turn facilitate the systematic study of proteins. In this study, we employed a more reliable analytical technique than those used in previous studies, to identify novel OS genes and understand disease mechanisms, by analyzing the behavior of modules across specific conditions in a controlled manner.

Here, we have successfully identified a total of 150 altered module pairs, as well as the 
important biological pathways of the intersection genes between altered modules and the PPI, with PCC $>1$. Moreover, we screened 6 key genes that may be related to OS. The results of RT-PCR and western blot analyses indicated that the expression of PCNA, ATP6V1G3, FEN1, $\mathrm{CDC} 7$, and RPA3 in OS samples differed significantly from that in normal controls; therefore, these were speculated to be potential markers of OS.

FEN1 is a protein-coding gene (Hiraoka et al., 1995), which encodes an essential enzyme in microhomology-dependent alternative end joining, an inaccurate pathway for the repair of doublestranded breaks in DNA (microhomology-mediated end joining) (Sharma et al., 2015). A previous study has reported that FEN1 mRNA and protein is differentially expressed between an OS and osteoblast cell line (Zhang et al., 2011). Therefore, we speculated that FEN1, a key regulator of the DNA damage response framework, could play a significant role in OS development.

The protein encoded by $\mathrm{CDC} 7, \mathrm{cdc} 7$ kinase, plays a major role in the regulation of cell cycle (Kim et al., 2003). CDC7 is conserved throughout eukaryotic evolution; that is, the majority of the eukaryotic cells produce $\mathrm{Cdc} 7$ kinase. It has been speculated that abnormal expression of this protein may be associated with neoplastic transformation in some tumors. For instance, it has been previously reported that the deletion of $\mathrm{CDC} 7$ abrogates the hydroxyureainduced activation of Chk1 in mouse embryonic stem cells and human cancer cell lines (Kim et al., 2008). Therefore, we concluded that CDC7 might also be an important biomarker of OS.

Through a systematic tracking of the altered modules from re-weighted normal and OS PPI networks, we conclude that the 5 genes identified in this study as key genes associated with the development of OS could be underlying biomarkers for the diagnosis and treatment of this tumor.

\section{Conflicts of interest}

The authors declare no conflict of interest.

\section{ACKNOWLEDGMENTS}

We would like to thank Dr. Wen Sun for critical reading the manuscript.

\section{REFERENCES}

Anninga JK (2013). Clinical and molecular features of high-grade osteosarcoma. Doctoral Thesis, Department of Paediatrics, Pathology, Bone Tumours, Faculty of Medicine, Leiden University Medical Center (LUMC), Leiden University.

Buddingh EP, Kuijjer ML, Duim RA, Bürger H, et al. (2011). Tumor-infiltrating macrophages are associated with metastasis suppression in high-grade osteosarcoma: a rationale for treatment with macrophage activating agents. Clin. Cancer Res. 17: 2110-2119. http://dx.doi.org/10.1158/1078-0432.CCR-10-2047

Gautier L, Cope L, Bolstad BM and Irizarry RA (2004). Affy - analysis of Affymetrix GeneChip data at the probe level. Bioinformatics 20: 307-315.http://dx.doi.org/10.1093/bioinformatics/btg405

Hiraoka LR, Harrington JJ, Gerhard DS, Lieber MR, et al. (1995). Sequence of human FEN-1, a structure-specific endonuclease, and chromosomal localization of the gene (FEN1) in mouse and human. Genomics 25: 220-225. http:// dx.doi.org/10.1016/0888-7543(95)80129-A

Huang W, Sherman BT and Lempicki RA (2009). Systematic and integrative analysis of large gene lists using DAVID bioinformatics resources. Nat. Protoc. 4: 44-57.http://dx.doi.org/10.1038/nprot.2008.211

Jones KB, Salah Z, Del Mare S, Galasso M, et al. (2012). miRNA signatures associate with pathogenesis and progression of osteosarcoma. Cancer Res. 72: 1865-1877. http://dx.doi.org/10.1158/0008-5472.CAN-11-2663

Kager L, Zoubek A, Pötschger U, Kastner U, et al.; Cooperative German-Austrian-Swiss Osteosarcoma Study Group (2003). Primary metastatic osteosarcoma: presentation and outcome of patients treated on neoadjuvant Cooperative Osteosarcoma Study Group protocols. J. Clin. Oncol. 21: 2011-2018. http://dx.doi.org/10.1200/JCO.2003.08.132 
Kaste SC, Pratt CB, Cain AM, Jones-Wallace DJ, et al. (1999). Metastases detected at the time of diagnosis of primary pediatric extremity osteosarcoma at diagnosis: imaging features. Cancer 86: 1602-1608. http://dx.doi.org/10.1002/ (SICI) 1097-0142(19991015)86:8<1602::AID-CNCR31>3.0.CO;2-R

Kim JM, Yamada M and Masai H (2003). Functions of mammalian Cdc7 kinase in initiation/monitoring of DNA replication and development. Mutat. Res. 532: 29-40. http://dx.doi.org/10.1016/j.mrfmmm.2003.08.008

Kim JM, Kakusho N, Yamada M, Kanoh Y, et al. (2008). Cdc7 kinase mediates Claspin phosphorylation in DNA replication checkpoint. Oncogene 27: 3475-3482. http://dx.doi.org/10.1038/sj.onc.1210994

Li M, Zhang H, Wang J-X and Pan Y (2012). A new essential protein discovery method based on the integration of proteinprotein interaction and gene expression data. BMC Syst. Biol. 6: 15.http://dx.doi.org/10.1186/1752-0509-6-15

Li ZM, Zhang HY, Wang YX and Wang WB (2016). MicroRNA-137 is downregulated in human osteosarcoma and regulates cell proliferation and migration through targeting FXYD6. J. Drug Target. 24: 102-110.

Liu G, Wong L and Chua HN (2009). Complex discovery from weighted PPI networks. Bioinformatics 25: 1891-1897. http://dx.doi.org/10.1093/bioinformatics/btp311

Liu S and Chen Z (2015). The functional role of PMP22 gene in the proliferation and invasion of osteosarcoma. Med. Sci. Monit. 21: 1976-1982. http://dx.doi.org/10.12659/MSM.893430

Luetke A, Meyers PA, Lewis I and Juergens H (2014). Osteosarcoma treatment - where do we stand? A state of the art review. Cancer Treat. Rev. 40: 523-532. http://dx.doi.org/10.1016/j.ctrv.2013.11.006

Ottaviani G and Jaffe N (2009). The epidemiology of osteosarcoma. Cancer Treat. Res. 152: 3-13. http://dx.doi. org/10.1007/978-1-4419-0284-9_1

Rosemann M, Gonzalez-Vasconcellos I, Domke T, Kuosaite V, et al. (2014). A Rb1 promoter variant with reduced activity contributes to osteosarcoma susceptibility in irradiated mice. Mol. Cancer 13: 182. http://dx.doi.org/10.1186/14764598-13-182

Sharma S, Javadekar SM, Pandey M, Srivastava M, et al. (2015). Homology and enzymatic requirements of microhomologydependent alternative end joining. Cell Death Dis. 6: e1697.http://dx.doi.org/10.1038/cddis.2015.58

Slonim DK (2002). From patterns to pathways: gene expression data analysis comes of age. Nat. Genet. 32 (Suppl): 502508. http://dx.doi.org/10.1038/ng1033

Srihari S and Leong HW (2013). A survey of computational methods for protein complex prediction from protein interaction networks. J. Bioinform. Comput. Biol. 11: 1230002.http://dx.doi.org/10.1142/S021972001230002X

Srihari S and Ragan MA (2013). Systematic tracking of dysregulated modules identifies novel genes in cancer. Bioinformatics 29: 1553-1561. http://dx.doi.org/10.1093/bioinformatics/btt191

von Mering C, Jensen LJ, Snel B, Hooper SD, et al. (2005). STRING: known and predicted protein-protein associations, integrated and transferred across organisms. Nucleic Acids Res. 33: D433-D437. http://dx.doi.org/10.1093/nar/ gki005

Zeng H and Xu X (2015). RUNX2 RNA interference inhibits the invasion of osteosarcoma. Oncol. Lett. 9: 2455-2458.

Zhang Y, Cai L, Wei RX, Hu H, et al. (2011). Different expression of alternative lengthening of telomere (ALT)-associated proteins/mRNAs in osteosarcoma cell lines. Oncol. Lett. 2: 1327-1332.

Genetics and Molecular Research 15 (3): gmr.15038277 